\title{
Optimization of magnetoelastic properties of pure nickel by means of heat treatments
}

\author{
A. L. Morales, A. J. Nieto, J. M. Chicharro, P. Pintado, \\ G. P. Rodríguez \& G. Herranz \\ Department of Applied Mechanics and Project Engineering, ETSII, \\ University of Castilla, La Mancha, Spain
}

\begin{abstract}
In this work we include valuable information about the way in which nickel may become a valuable smart material for some applications and we provide a better understanding of the influence of internal stresses on its magnetoelastic effects. The different states of internal stress are achieved via different heat treatments obtained by modifying three main parameters: the heating temperature, the heating time and the cooling method. Then, we carried out the next works: first, a microscopic analysis of the grain size of the samples under different heat treatments; second, all the tested specimens were subjected to an exhaustive internal stress analysis via X-Ray diffraction techniques; and third, the $\Delta \mathrm{E}$ - and $\Delta \Psi$-effects were estimated in order to link the internal stress state of the sample to its magnetoelastic response. The results can guide us in selecting the most suitable heat treatment in order to make nickel show the smart properties we desire.

Keywords: magnetoelasticity, internal stress, heat treatments, nickel, X-Ray diffraction.
\end{abstract}

\section{Introduction}

Magnetomechanical materials are a kind of smart material in which there is a reciprocal coupling between their mechanical and magnetic properties [1]. In this broad category, described by Jiles in [2], we can find both magnetoelastic and magnetoplastic materials. The former are those in which the variation of the altered properties is reversible, whereas the latter are those in which the recovery of the properties is not necessarily obtained simply on removal of the magnetic or mechanical action. 
Some crystalline pure nickel results about $\Delta \mathrm{E}$-effect and magnetomechanical damping were previously published in our recent work [3]. In said work, nickel clearly stands out from the rest of classical ferromagnetic materials like iron and cobalt, but yet without showing enough variations to be considered a suitable choice for any application. A bit later, we published our work [4], which also deals with the influence of internal stresses in the magnetoelastic behaviour of nickel and provides a great amount of significant quantitative data related to this topic. In the present work, not only do we want to provide further results, details and discussions related to the microscopic and internal stress characterization of this material, but also we include a qualitative discussion about the way in which this comprehensive characterization can help us to optimize its magnetoelastic response in accordance to different objective functions.

Other works such as [5-7] have also dealt with this topic but they considered either different effects or different materials. In this work in particular, two salient magnetoelastic effects will be measured in order to analyze the influence of internal stress on magnetoelasticity in nickel: the dependence of elastic modulus and damping on the magnetic field, i.e., the so-called $\Delta \mathrm{E}-$ and $\Delta \Psi$ effects. The interested reader may find a theoretical background about these effects and the influence of internal stress on them in several works such as [8].

\section{Experimental details}

Pure crystalline rods of nickel 201, all from the same molten material and manufacturing process, were used in this work. They were $110 \mathrm{~mm}$ in length and $10 \mathrm{~mm}$ in diameter and they showed a purity level of $99.90 \%$ and a density of $8912 \mathrm{~kg} / \mathrm{m}^{3}$.

The magnetoelastic properties were measured via a recently experimental system which we developed in [9] and enhanced later in [3]. It is based on laser Doppler vibrometry and it turns out to be a suitable method for measuring $\Delta \mathrm{E}$ and $\Delta \Psi$ - effects: not only is it able to measure both effects simultaneously and accurately, but it also allows us to obtain stress- and path-dependencies.

The different heat treatments are achieved by modifying three main parameters: the heating temperature, the heating time and the cooling method. The selection of the different heating temperatures for our scheduled heat treatments, probably the more significant parameter from the point of view of internal stresses, was made in accordance with the recommendations found in [10] for nickel specimens. This led us to three different treatments: annealing $\left(705-1205^{\circ} \mathrm{C}\right)$, designed to produce a recrystallized grain structure and softening in work-hardened alloys; stress relieving $\left(425-870^{\circ} \mathrm{C}\right)$, used to reduce stresses in work-hardened non-age-hardenable alloys without recrystallizing the grain structure; and stress equalizing $\left(230-315^{\circ} \mathrm{C}\right)$, used to balance stresses in coldworked materials without appreciably decreasing mechanical strength. Table 1 presents a summary of the heat treatments. In it, each specimen has been identified by three digits (which represent the heating temperature in Celsius), two digits (which indicate the heating time in hours) and one letter (which refers 
to the cooling method, i.e., water, air or furnace). The initial state of the nickel specimens is labelled "AR" (as received).

Finally we want to emphasize that, leaving aside field-dependence, other influences such as stress-dependence and path-dependence must be considered in our measurements [3]. In order to avoid their influences, we will report only those results which were obtained under $0.50 \mathrm{MPa}$ and following a magnetization path from the state of zero retentivity $\left(\mathrm{M}_{\mathrm{r}}{ }^{0}\right)$ to the state of positive saturation magnetization $\left(\mathrm{M}_{\mathrm{s}}^{+}\right)$.

Table 1: $\quad$ Scheduled heat treatments.

\begin{tabular}{|c|c|c|c|c|}
\hline Specimen & $\begin{array}{c}\text { Heating } \\
\text { temp. }\left({ }^{\circ} \mathrm{C}\right)\end{array}$ & $\begin{array}{c}\text { Heating time } \\
(\mathrm{h})\end{array}$ & $\begin{array}{c}\text { Cooling } \\
\text { method }\end{array}$ & Nomenclature \\
\hline 0 & - & - & - & AR \\
\hline 1 & 300 & 2 & Water & $300-02-\mathrm{W}$ \\
\hline 2 & 300 & 2 & Air & $300-02-\mathrm{A}$ \\
\hline 3 & 600 & 2 & Water & $600-02-\mathrm{W}$ \\
\hline 4 & 600 & 2 & Air & $600-02-\mathrm{A}$ \\
\hline 5 & 900 & 2 & Water & $900-02-\mathrm{W}$ \\
\hline 6 & 900 & 2 & Air & $900-02-\mathrm{A}$ \\
\hline 7 & 900 & 2 & Furnace & $900-02-\mathrm{F}$ \\
\hline 8 & 900 & 4 & Water & $900-04-\mathrm{W}$ \\
\hline 9 & 900 & 4 & Air & $900-04-\mathrm{A}$ \\
\hline 10 & 900 & 4 & Furnace & $900-04-\mathrm{F}$ \\
\hline 11 & 900 & 8 & Water & $900-08-\mathrm{W}$ \\
\hline 12 & 900 & 8 & Air & $900-08-\mathrm{A}$ \\
\hline 13 & 900 & 8 & Furnace & $900-08-\mathrm{F}$ \\
\hline
\end{tabular}

\section{Results and discussion}

\subsection{Microscopic analysis of grain size}

The scheduled heat treatments were carried out in a Carbolite Eurotherm 2416 furnace. The preparation procedure for microscopic examination of the specimens was made in accordance to [11]. In particular, the specimens were cut with a precision Struers Secotom-10 saw, then mounted in a hard epoxy resin with a Struers LaboPress-3 mounting press and finally ground and polished in a Struers TegraSystem machine (TegraPol-15 and TegraForce-1). The etchant applied to the surfaces was a fresh solution of one part $\mathrm{HNO}_{3}$ and one part acetic acid (glacial), which is the recommended etchant for revealing grain boundaries in nickel 201. For the microscopic examination, the equipment used was a Leica DM IRM inverted research microscope with a Leica DFC 480 high performance digital FireWire camera system which allowed us to obtain micrographies and estimations of the grain size in each specimen. Fig. 1 shows the micrographies for the 8 most significant specimens. 

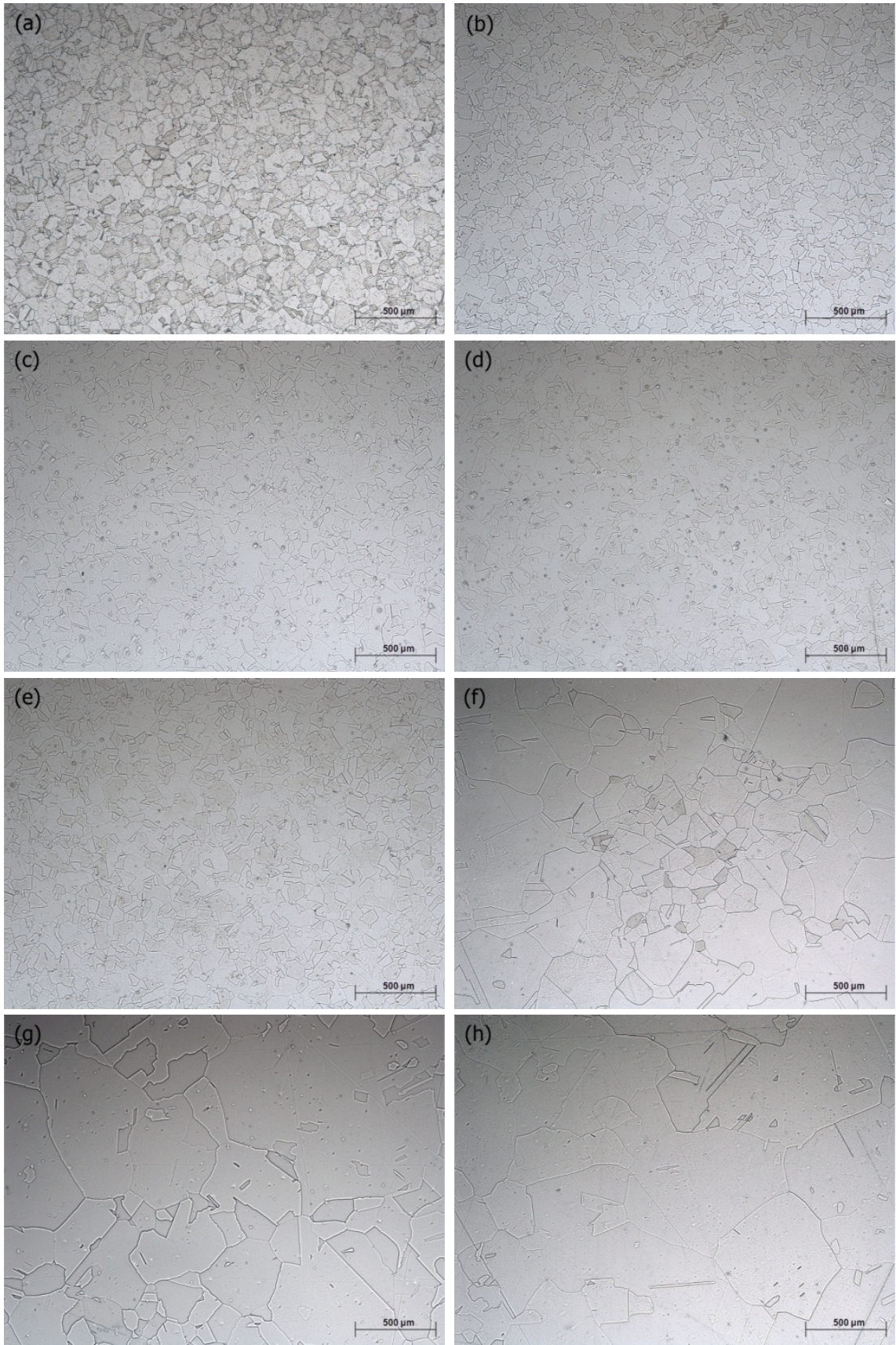

Figure 1: $\quad$ Micrographies of specimens (a) AR, (b) 300-02-W, (c) 300-02-A, (d) 600-02-W, (e) 600-02-A, (f) 900-02-W, (g) 900-02-F and (h) 900-08-W.

WIT Transactions on Engineering Sciences, Vol 72, (C) 2011 WIT Press www.witpress.com, ISSN 1743-3533 (on-line) 
On the one hand, specimens which were subjected to stress relieving (300-02$\mathrm{W}$ and 300-02-A) and stress equalizing (600-02-W and 600-02-A) processes show a grain size similar to that of specimen AR. This fact agrees with the fact that stress relieving and stress equalizing reduce and balance stresses without recrystallizing the grain structure. Besides, in spite of small variations, we can also observe higher grains in specimens which were subjected to stress equalizing due to the higher heating temperature and consequently the higher grain boundary diffusion. On the other hand, annealed specimens (900-02-W, 900-02-F and 900-08-W) clearly shows much larger grains, which will translate into lower internal stresses. Specimen 900-08-F has not been shown because it has got almost the same distribution of grains due to the long heating time, which means high grain boundary diffusion even when cooled in water. Furthermore, the concentration of small grains which appears in specimen 900-02-W corresponds to the centre of the specimen, where the heating time was not high enough to continue increasing the size of inner grains (the diffusion processes goes from the outside to the inside of the rod).

Table 2: $\quad$ Microscopic measurements.

\begin{tabular}{|c|c|c|l|l|c|c|}
\hline \multirow{2}{*}{ Specimen } & \multicolumn{2}{|c|}{ Small grains } & \multicolumn{2}{l|}{ Medium grains } & \multicolumn{2}{c|}{ Large grains } \\
\cline { 2 - 7 } & $\begin{array}{c}\text { Perim. } \\
(\mu \mathrm{m})\end{array}$ & $\begin{array}{c}\text { Area } \\
\left(\mu \mathrm{m}^{2}\right)\end{array}$ & $\begin{array}{l}\text { Perim. } \\
(\mu \mathrm{m})\end{array}$ & $\begin{array}{l}\text { Area } \\
\left(\mu \mathrm{m}^{2}\right)\end{array}$ & $\begin{array}{c}\text { Perim. } \\
(\mu \mathrm{m})\end{array}$ & $\begin{array}{c}\text { Area } \\
\left(\mu \mathrm{m}^{2}\right)\end{array}$ \\
\hline AR & 350 & 8200 & - & - & - & - \\
\hline $300-02-\mathrm{W}$ & 440 & 11900 & - & - & - & - \\
\hline $300-02-\mathrm{A}$ & 520 & 14400 & - & - & - & - \\
\hline $600-02-\mathrm{W}$ & 600 & 16900 & 1240 & 41000 & - & - \\
\hline $600-02-\mathrm{A}$ & 700 & 21500 & 1240 & 53000 & - & - \\
\hline $900-02-\mathrm{W}$ & 530 & 16600 & 1720 & 144000 & 4000 & 590000 \\
\hline $900-02-\mathrm{A}$ & 620 & 20700 & 1730 & 157000 & 4200 & 510000 \\
\hline $900-02-\mathrm{F}$ & 620 & 23600 & 1660 & 147000 & 4100 & 650000 \\
\hline $900-04-\mathrm{W}$ & - & - & 1640 & 137000 & 4200 & 670000 \\
\hline $900-04-\mathrm{A}$ & - & - & 1820 & 167000 & 4400 & 670000 \\
\hline $900-04-\mathrm{F}$ & - & - & 1750 & 143000 & 4700 & 660000 \\
\hline $900-08-\mathrm{W}$ & - & - & 1960 & 194000 & 5300 & 780000 \\
\hline $900-08-\mathrm{A}$ & - & - & 1900 & 195000 & 5300 & 720000 \\
\hline $900-08-\mathrm{F}$ & - & - & 1870 & 170000 & 5600 & 940000 \\
\hline
\end{tabular}

Table 2 shows the measurements of grain perimeter and grain area for each specimen. The results seemed to indicate the existence of three different distributions: small grains, with a perimeter lower than $1000 \mathrm{~mm}$; medium grains, with a perimeter between 1000 and $3000 \mathrm{~mm}$; and large grains, with a perimeter greater than $3000 \mathrm{~mm}$. Specimens AR, 300-02-W and 300-02-A only contain small grains, although perimeters and areas increase slightly when the specimens have been heated. Specimens 600-02-W and 600-02-A show small grains mainly although medium grains are not uncommon. Finally, all specimens heated to $900^{\circ} \mathrm{C}$ show large distributions of medium and large grains due to 
recrystallization, whereas small grains tend to disappear (apart from the previously mentioned significant distribution of small grains in the centre of the section of specimens which were subjected to a time of only 2 hours). In general, the grain size increases with heating time and decreases with cooling velocity.

\subsection{Internal stress analysis}

Internal or residual stress is an extrinsic property which cannot be measured directly but through the previous measurement of an intrinsic property of the material. In this particular case, we have used the strain in the crystal lattice as the intrinsic variable which will allow us to estimate the residual stresses of our specimens. Among all the ways to measure the strain in the crystal lattice, the XRay diffraction technique clearly stands out.

$\mathrm{X}$-Ray diffraction techniques can be used to determine both macroscopic (homogeneous) and microscopic (inhomogeneous) residual stresses [12, 13]. In this work we only take into account microstresses, which are those involved in cold-work, plastic strains or heat treatments and lead to the accumulation of a residual dislocation network, producing inhomogeneous strain and an irreversible broadening of the Bragg peaks in X-Ray diffraction. Indeed, the measurement of said broadening of the diffraction peak will be used to estimate the internal stresses of our specimens.

Strictly speaking, we have used the Williamson-Hall method [12], which has proven its validity to estimate residual microstresses remaining in materials after heat treatments [13]. The X-Ray diffraction profiles (intensity vs. angle of diffraction) have been recorded using a PHILIPS X'Pert MPD X-Ray diffractometer with $\mathrm{CuKa}$ radiation (wavelength $\lambda=1,5405 \AA$ ) and varying the angle of diffraction $(\theta)$ from $40^{\circ}$ to $100^{\circ}$ with a scan step of $0.04^{\circ}$ and a time per step of 5 seconds. Fig. 2 and Fig. 3 show, respectively, the X-Ray full diffraction profile for the specimen AR and the fitting procedure of one peak of the X-Ray diffraction profile for the specimen $900-02-\mathrm{W}$. No other plots are included since the peaks position in all the specimens remain the same and the variation in width is not high enough to be easily noticed at a glance.

The width of a peak can be calculated via two different ways: as the integral breadth $\beta$, defined as the width of a rectangle having the same area and height as the observed line profile, or as the full width at half maximum FWHM. WinPLOTR software allowed us to obtain both of them for several reflections as a measure of the peak width, but we only used the integral breadth in our estimations. The strain calculated using WinPLOTR is the so-called maximum strain.

Besides, a diffraction pattern has contributions from the instrument optics (even a perfect sample will give lines of finite width), from particle size and from microstrain. The way in which these contributions are added depends on the shape of the peak. In our case, we assume that peaks are Lorentzian in shape, so all these contributions can be directly added:

$$
\beta_{\text {exp }}=\beta_{\text {inst }}+\beta_{\text {size }}+\beta_{\text {strain }}
$$




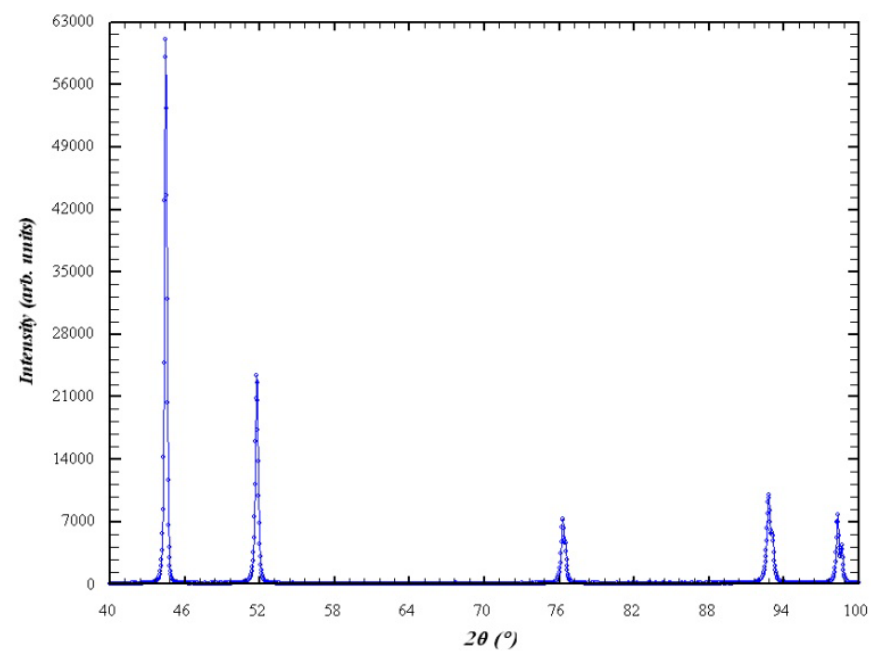

Figure 2: X-Ray diffraction profile for the specimen AR obtained in the software WinPLOTR.

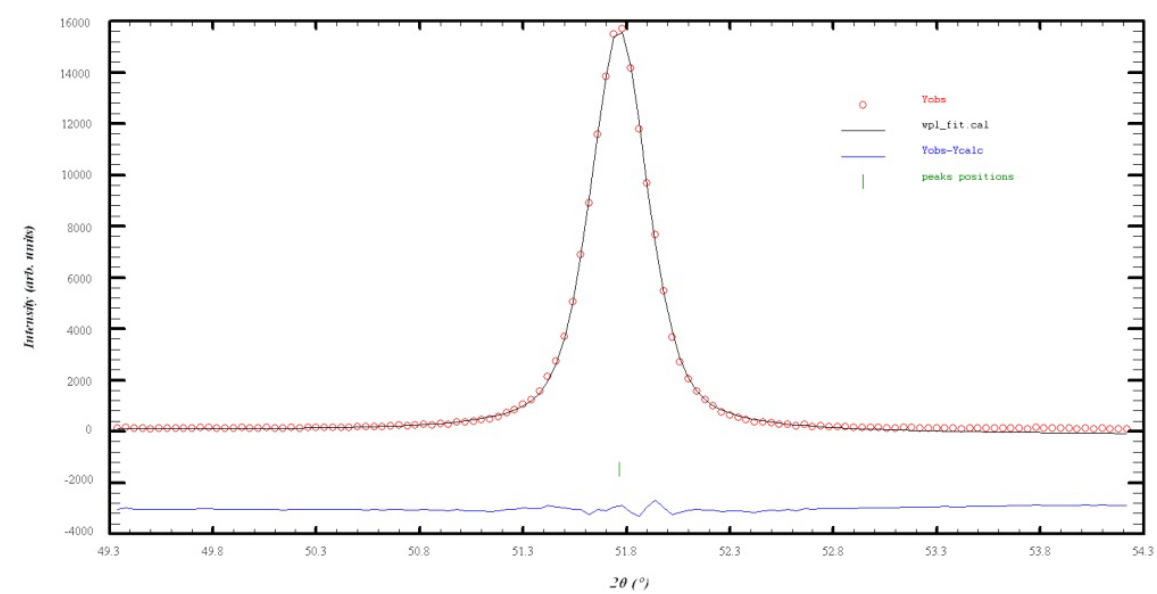

Figure 3: Detail of the second peak of the X-Ray diffraction profile for the specimen $900-02-\mathrm{W}$ and the fitting procedure in WinPLOTR.

Now, considering that we can make the correction for the instrumental broadening, the corrected integral breath due to microstrain and particle size can be written as follows:

$$
\beta_{\text {corr }}=\frac{0.9 \lambda}{t \cos \theta}+\frac{4 \varepsilon}{\tan \theta}
$$




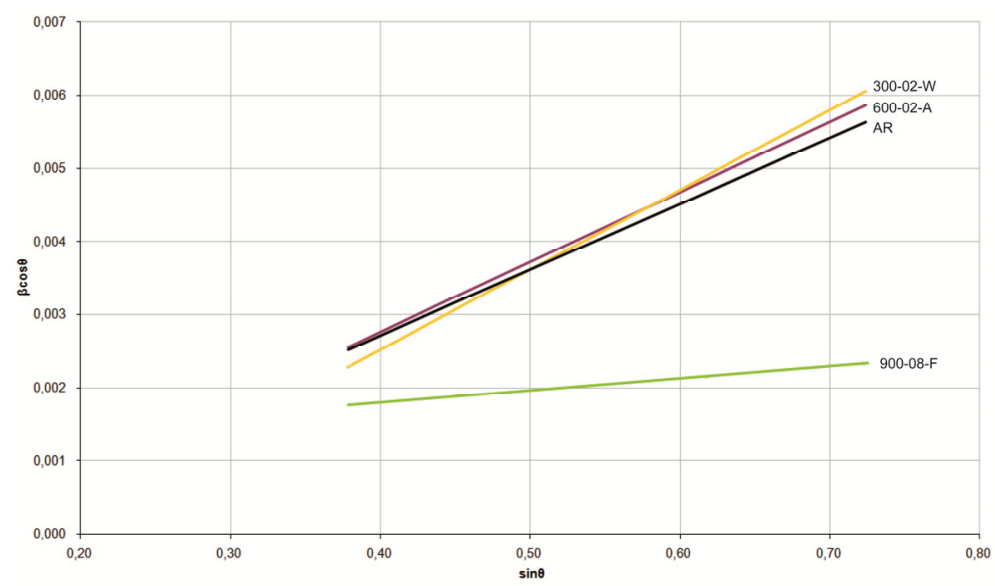

Figure 4: Williamson-Hall plots of specimens AR, 300-02-W, 600-02-A and 900-08-F.

where the first addend is the particle size contribution and the second addend is the strain $(\varepsilon)$ contribution. Then, left-multiplying by $\cos \theta$ we obtain:

$$
\beta_{\text {corr }} \cos \theta=\frac{0.9 \lambda}{t}+4 \varepsilon \sin \theta
$$

This expression leads to the well-known Williamson-Hall plot [12], which is the plot of $\beta \cos \theta$ against $\sin \theta$. Thus, the strain is directly related to the slope of said plot. Fig. 4 shows some selected Williamson-Hall plots.

Table 3 gathers all the internal stresses obtained from the Williamson-Hall plots. In addition, microstrain, demagnetized elastic modulus and lattice parameter have been also included, this latter being known very easily once we know the wavelength of the radiation and the angle of diffraction of each peak.

Regarding internal stresses, results provided in Table 3 are in good agreement with what one would expect given the heat treatments carried out. Indeed, we can distinguish three different ranges of residual stresses:

- High residual stresses (over $500 \mathrm{MPa}$ ). These values are shown by the specimens which were subjected to stress equalizing $\left(230-315^{\circ} \mathrm{C}\right.$ and air or furnace cooling [10]). Water quenching was also tested obtaining, as expected, the highest value.

- Medium residual stresses (between 300 and $500 \mathrm{MPa}$ ). These values correspond to the specimen AR and to those which have been subjected to stress relieving treatments $\left(425-870^{\circ} \mathrm{C}\right.$ and air or furnace cooling $[10])$. As the name of the treatment indicates, a small stress reduction is achieved.

- Low residual stresses (below $300 \mathrm{MPa}$ ). These values are shown by samples that have been subjected to annealing process $\left(705-1205^{\circ} \mathrm{C}\right.$ and air or furnace cooling [10]). In these cases, a high reduction of internal stresses is achieved. Nevertheless, if water quenching is applied, the internal stresses jump into a level of medium stresses. 
Regarding the lattice parameter, no significant variations were found, but the obtained value is in good agreement with the typical values of the lattice parameter in nickel samples (around $3.5 \AA$ ).

Table 3: Internal stresses and lattice parameter.

\begin{tabular}{|c|c|c|c|c|}
\hline Specimen & $\begin{array}{c}\text { Internal } \\
\text { stress (MPa) }\end{array}$ & $\begin{array}{c}\text { Microstrain } \\
(\%)\end{array}$ & $\begin{array}{c}\text { Elastic } \\
\text { modulus }(\mathrm{GPa})\end{array}$ & $\begin{array}{c}\text { Lattice } \\
\text { parameter }(\AA)\end{array}$ \\
\hline AR & 479.0 & 0.23 & 213 & 3.53 \\
\hline $300-02-\mathrm{W}$ & 579.6 & 0.27 & 213 & 3.53 \\
\hline $300-02-\mathrm{A}$ & 536.6 & 0.25 & 213 & 3.53 \\
\hline $600-02-\mathrm{W}$ & 475.2 & 0.22 & 216 & 3.53 \\
\hline $600-02-\mathrm{A}$ & 498.3 & 0.24 & 210 & 3.53 \\
\hline $900-02-\mathrm{W}$ & 469.0 & 0.22 & 220 & 3.53 \\
\hline $900-02-\mathrm{A}$ & 276.9 & 0.14 & 201 & 3.54 \\
\hline $900-02-\mathrm{F}$ & 223.4 & 0.11 & 199 & 3.53 \\
\hline $900-04-\mathrm{W}$ & 356.6 & 0.17 & 213 & 3.54 \\
\hline $900-04-\mathrm{A}$ & 248.6 & 0.13 & 203 & 3.54 \\
\hline $900-04-\mathrm{F}$ & 106.8 & 0.05 & 203 & 3.54 \\
\hline $900-08-\mathrm{W}$ & 415.8 & 0.20 & 216 & 3.52 \\
\hline $900-08-\mathrm{A}$ & 207.6 & 0.10 & 208 & 3.53 \\
\hline $900-08-\mathrm{F}$ & 86.2 & 0.04 & 203 & 3.53 \\
\hline
\end{tabular}

\section{3 $\Delta \mathbf{E}-$ and $\Delta \Psi$-effects}

The results corresponding to the $\Delta \mathrm{E}$ - and $\Delta \Psi$-effects of the 14 nickel specimens considered show similarities which can be summarized mainly into two different patterns which are depicted in Fig. 5 and explained next:

- $\quad$ Pattern I is shown by specimen AR and all those which were subjected to heat treatments of stress relieving and stress equalizing. Indeed, specimens subjected to a heating temperature of $900^{\circ} \mathrm{C}$ and water cooled also may be grouped into this patter. On the one hand, the curve of elastic modulus is compound by an initial stage of rapid growth which belongs to the low magnetic field range (less than $150 \mathrm{Oe}$ ) and a second stage of slow growth until saturation (around $350 \mathrm{Oe}$ ). On the other hand, the curve of damping shows again two stages, an initial rising stage which corresponds to the low applied magnetic field range and the second declining stage until saturation. In both cases, variations between the values at demagnetized and saturated states are lower than those found in pattern II.

- $\quad$ Pattern II is shown by the specimens which were heated up to $900^{\circ} \mathrm{C}$ and slowly cooled down in air or inside the furnace, so they present a very low level of internal stresses. The elastic modulus curves show the same behaviour as in pattern I but variations between the values at demagnetized and saturated states are greater. As far as the damping curves are concerned, they show a very high damping value which quickly start to decrease until saturation without intermediate peaks. 

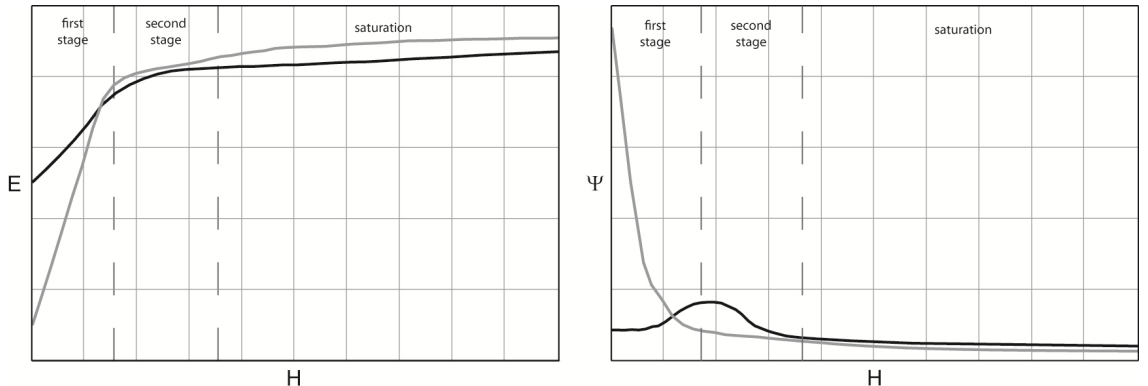

Figure 5: Different patterns (I: black; II: gray) of the $\Delta \mathrm{E}$ - and $\Delta \Psi$ - effects.

(a)

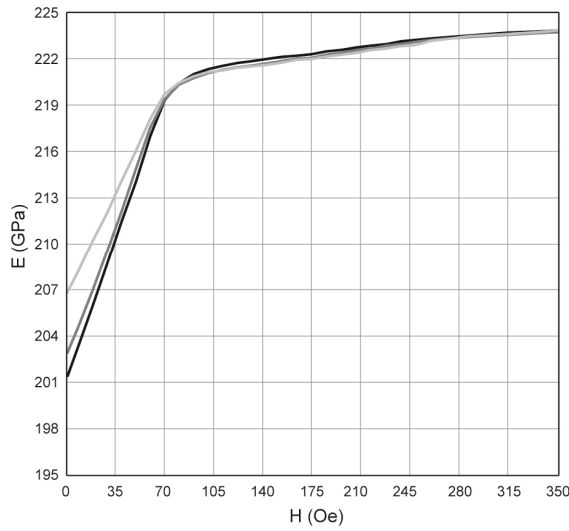

(b)

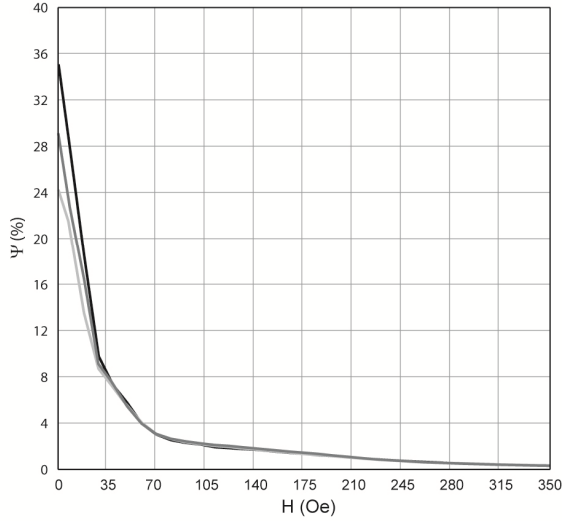

Figure 6: Influence of heating time on (a) $\Delta \mathrm{E}$ - and (b) $\Delta \Psi$ - effects (black: 900-02-A; dark gray: 900-04-A; light gray: 900-08-A).

Since it is not the main goal of this work, we will not include results about the $\Delta \mathrm{E}$ - and $\Delta \Psi$ - effects of each treated specimen. The interested reader may consult such results in our work [4].

Regarding the influence of the parameters involved in the heat treatments, we can claim than the heating temperature is the most significant parameter since only temperatures higher than $700^{\circ} \mathrm{C}\left(900^{\circ}\right.$ in our tests) may lead to a pattern II behaviour. The cooling methods also stand out as a significant parameter since water cooling may avoid the grain diffusion and increase internal stresses, what would lead to a pattern I behaviour. Finally, the heating time has a very minute influence on the magnetoelastic behaviour when using long cooling methods such as air cooling or furnace cooling. This can be seen in Fig. 6.

Another important feature we should analyze is the influence of the heat treatments and the internal stresses on the magnetic field from which the elastic modulus or the magnetomechanical damping remains the same, i.e., on the magnetic field which makes the $\Delta \mathrm{E}$ - and $\Delta \Psi$ - effects saturate. We can conclude that in all the cases the saturating magnetic field is around $350 \mathrm{Oe}$, so it does not depend on any of the heat treatment parameters which were considered. 


\section{Conclusions}

The previous results can guide us in selecting the most suitable heat treatment in order to make nickel show the smart properties we desire. It is important to notice that the optimization will depend on the objective function we select. Smart materials are usually required to provide a maximum variation of the salient magnitude by supplying a minimum modification of the input. This fact, from the point of view of a magnetoelastic material, means large variations in applied forces, natural frequencies and damping ratios obtained via small changes in the applied magnetic field. Nevertheless, we should not discard that, in some cases, the objective is the opposite: to obtain a constant value of elastic modulus and damping along a range of magnetic field as large as possible.

We can extract valuable information about the different possibilities of optimization from Fig. 5. On the one hand, if nickel is required to act as a smart material which provides the highest $\Delta \mathrm{E}$ - and $\Delta \Psi$ - effects, then it should behave following pattern II. In addition, the characteristic damping peak of pattern I disappears, so we get a more predictable behaviour. Thus, in the light of section 3 results, the material should show very low internal stresses, which can be achieved via annealing heat treatments with slow cooling velocities (900-0X-A or 900-0X-F). On the other hand, one may need that nickel show elastic and damping properties as constant as possible under different applied magnetic fields. Although this behaviour cannot be perfectly fitted, it is possible to minimize the $\Delta \mathrm{E}$ - and $\Delta \Psi$ - effects by making the material follow pattern $\mathrm{I}$. Again, we know from discussion in section 3, that this means getting internal stresses as high as possible, which can be achieved via work-hardening, stress equalizing heat treatments and fast cooling velocities (AR, 300-0X-W).

Leaving aside optimization rules, this work also provides relevant results, details and discussions related to the microscopic and internal stress characterization of this material. We have carried out an in-depth material characterization in terms of grain distribution and internal stresses when nickel specimens are subjected to several different heat treatments. On the one hand we have included many micrographies which help us to understand not only the way does each heat treatment modify the grain size, but also how the grain diffusion acts depending on the heating temperature, the heating time and the cooling method. On the other hand we have carefully described the way we have obtained the microstrain, internal stress and lattice parameter in all our specimens via X-Ray diffraction and the Williamson-Hall method. All these results agree with the fact that the internal stress decreases when we increase the heating temperature and decrease the cooling velocity, the heating time becoming an almost negligible parameter.

\section{References}

[1] du Trémolet de Lacheisserie, E., Magnetostriction: Theory and Applications of Magnetoelasticity, CRC Press: Boca Raton, 1993. 
[2] Jiles, D.C., Recent advances and future directions in magnetic materials, Acta Materialia, 51, pp. 5907-5939, 2003.

[3] Morales, A.L., Nieto, A.J., Chicharro, J.M. \& Pintado, P., Field-dependent elastic modulus and damping in pure iron, nickel and cobalt, Journal of Magnetism and Magnetic Materials, 322, pp. 1952-1961, 2010.

[4] Morales, A.L., Nieto, A.J., Chicharro, J.M. \& Pintado, P., Influence of internal stresses on field-dependent elastic modulus and damping in pure nickel, Journal of Magnetism and Magnetic Materials, 322, pp. 3584-3594, 2010 .

[5] Motogi, S. \& Maugin, G.A., Elastic-moduli of demagnetized polycrystalline ferromagnets, Journal of Physics D-Applied Physics, 26, pp. 1459-1467, 1993.

[6] Squire, P.T., Atkinson, D., Gibbs, M.R.J. \& Atalay, S., Amorphous wires and their applications, Journal of Magnetism and Magnetic Materials, 132, pp. 10-21, 1994.

[7] Kaczkowski, Z., Magnetomechanical properties of rapidly quenched materials, Materials Science and Engineering A-Structural Materials Properties Microstructure and Processing, 226-228, pp. 614-625, 1997.

[8] Bozorth, R.M., Ferromagnetism, D. van Nostrand: Toronto, 1951.

[9] Morales, A.L., Nieto, A.J., Chicharro, J.M. \& Pintado, P., Automatic measurement of field-dependent elastic modulus and damping by laser Doppler vibrometry, Measurement Science and Technology, 19, 2008.

[10] International ASM, ASM Handbook Vol. 4-Heat Treating, vol. 4, ASM International, 1991.

[11] International ASM, ASM Handbook Vol. 9-Metallography and Microstructures, vol. 9, ASM International, 2004.

[12] Williamson, G.K. \& Hall, W.H., X-Ray line broadening from filed aluminium and wolfram, Acta Metallurgica, 1, pp. 22-31, 1953.

[13] Prevéy, P.S., The measurement of subsurface residual stress and cold work distributions in nickel base alloys, ASM's Conference on Residual Stress in Design, Process and Materials Selection, ASM International, Cincinnati (Ohio, USA), pp. 27-29, 1987. 